\title{
Radiative transfer simulations of multiphase AGN tori: thermal emission and polarisation
}

\author{
M. Baes ${ }^{1}$, M. Stalevski ${ }^{1,2,3}$, P. Camps ${ }^{1}$, J. Fritz ${ }^{4}$ and L. Č. Popović ${ }^{3}$ \\ ${ }^{1}$ Sterrenkundig Observatorium, Universiteit Gent, Belgium \\ ${ }^{2}$ Departamento de Astronomía, Universidad de Chile, Chile \\ ${ }^{3}$ Astronomical Observatory, Belgrade, Serbia \\ ${ }^{4}$ Centro de Radioastronomía y Astrofísica, UNAM Morelia, Mexico
}

\begin{abstract}
The unification model of active galactic nuclei postulates an accreting supermassive black hole as the central engine, surrounded by a putative dusty torus. This dust absorbs the incoming radiation, re-emits it in the infrared and obscures our view of the central region at certain inclinations. We present a new set of AGN models, in which the torus is modelled as a 3D multiphase medium. These new models can explain the observed spectral energy distribution of AGNs over the entire infrared domain, including the observed silicate feature strength and the level of near-infrared continuum. A new generation of multi-phase models, based on hydrodynamical simulations, is being constructed. We will compute the polarisation structure of these physically motivated $3 \mathrm{D}$ torus models, and compare them to simpler smooth torus models and to the available observational data.
\end{abstract}

Keywords. galaxies: active, radiative transfer, radiation mechanisms: thermal, polarisation

\section{Introduction}

Active galactic nuclei (AGNs) can be subdivided in two broad classes: type 1 AGNs with broad emission lines and a strong UV/optical emission, and type 2 AGNs, characterised by narrow emission lines and lacking the UV/optical bump. According to the AGN unification model, different appearances of type 1 and type 2 AGNs are only a matter of orientation (Antonucci 1993, Urry \& Padovani 1995). This broadly accepted hypothesis postulates that the black hole and its accretion disc are surrounded by a thick, roughly toroidal structure of dust and gas. This unified model is supported by a variety of observational evidence, including spectropolarimetric measurements, the presence of ionising cones and the distribution of X-ray absorption. While the existence of a dusty obscuring torus around AGNs is nowadays generally accepted, the structural properties (extent, elongation, geometry,...), formation and evolution of this torus are still very uncertain.

\section{Multiphase AGN torus models}

In the past three decades, the strongest constraints on the geometry of AGN tori have been obtained using radiative transfer models that self-consistently reproduce the observed infrared emission (the dust in the torus absorbs the UV/optical emission from the accretion disc and reemits the absorbed energy in the infrared). The first generation of AGN torus models was characterised by a smooth and continuous dust distribution (e.g., Efstathiou \& Rowan-Robinson 1995; Schartmann et al. 2005; Fritz et al. 2006). Such models were in general able to reproduce the observed spectral energy distribution (SED) of both local and distant AGNs, but reproducing the intensity and the shape of the mid-infrared silicate features turned out to be a major challenge of the smooth AGN 
$4 \mu \mathrm{m}$

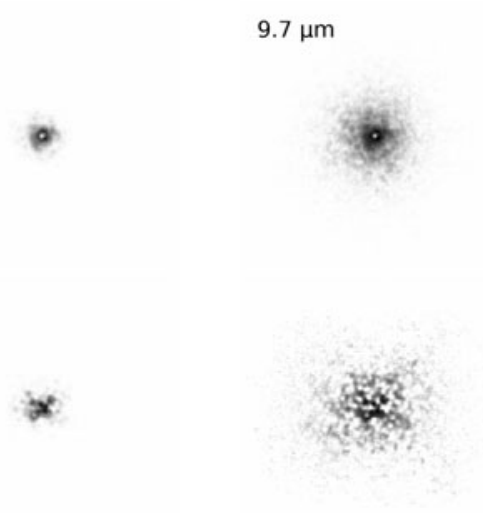

$13.9 \mu \mathrm{m}$
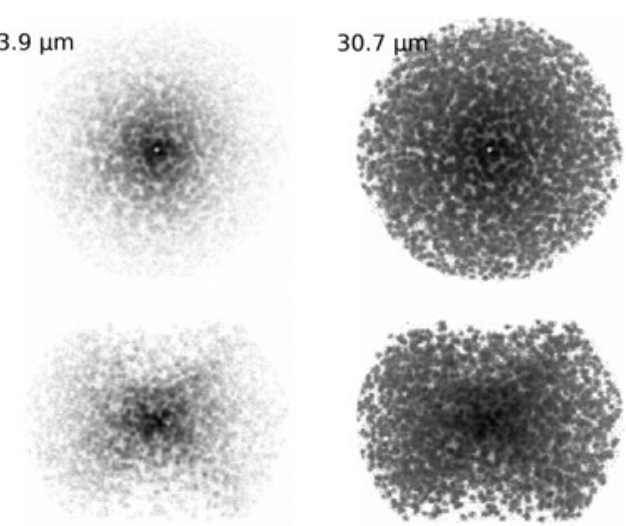

Figure 1. Images of an example of a two-phase torus model from Stalevski et al. (2012a), seen at different wavelengths. Top row is face-on view, bottom row is edge-on view. From left to right, panels represent images at 4.6, 9.7, 13.9 and $30.7 \mu \mathrm{m}$. Images are given in logarithmic colour scale.

models. A second generation of models had the dust located in a large number of optically thick clumps (e.g, Nenkova et al. 2002, 2008a, 2008b; Dullemond \& van Bemmel 2005; Hönig et al. 2006; Schartmann et al. 2008). Such models seemed to lack a hot emission component, resulting in an underestimate of the observed near-infrared continuum emission. In general, significant differences are present between the various clumpy torus models that have been developed (for a comparison between smooth and clumpy models, see Feltre et al. 2012).

We present a new set of AGN models, where we model the dusty torus as a 3D twophase medium with high-density clumps and low-density medium filling the space between the clumps (Stalevski et al. 2012a, see Fig. 1). By arranging the location of the clumps, the density structure corresponds to a complex structure of merged clumps or an interconnected sponge-like structure. The calculations were performed with SKIRT, a 3D dust radiative transfer code based on the Monte Carlo technique (Baes et al. 2003, 2011; Camps \& Baes 2015). The dust distribution, optical depth, clump size and the actual arrangement of the clumps in the innermost region all have an impact on the shape of the infrared SED. The $9.7 \mu \mathrm{m}$ silicate feature can display the entire range of strengths covered by observations. Moreover, these models offer a natural solution to the lack of near-infrared emission that is often present in clumpy models. These two-phase AGN torus models are incorporated in the SKIRTOR library (Stalevski 2012), and have been used in studies of gravitational microlensing (Stalevski et al. 2012b) and photometric variability (Popović et al. 2012).

We are now constructing even more realistic multi-phase geometries for AGN tori with SKIRT, based on the output of the hydrodynamic simulations as input for our models. Dust radiative transfer calculations based on such general and complex 3D geometries are now possible thanks to the use of advanced grids that can adapt their resolution where needed (Saftly et al. 2013, 2014; Camps et al. 2013). Preliminary tests based on the hydrodynamical simulations from Wada et al. (2009) are promising (see also Schartmann et al. 2014). 


\section{Polarisation as an additional constraint}

As the central structure of AGNs cannot be resolved (unless with interferometry), we need to use all options to compare the models to observational data in order to constrain the geometry. Scattered light is polarised and the angle of polarisation depends on the direction of the last scattering, so it is expected to be related to the structure of different emitting and scattering regions. As the radiation of AGNs is polarised over a broad wavelength range, the polarisation can be exploited to constrain their structure and geometry. UV and optical (spectro)polarimetry data are available for many nearby AGNs (Smith et al. 2002; Batcheldor et al. 2011; Marin 2014).

We are currently implementing polarisation due to scattering off dust grains and electrons into SKIRT. The first comparisons to the STOKES code (Marin et al. 2012; Goosmann et al. 2014) are encouraging. Once polarisation is fully operational in SKIRT, we will compute the polarisation properties of our complex and physically motivated 3D torus models. These will be used to perform a comparison at two different levels. First, we will calculate the polarisation properties and compare them to those corresponding to the smooth, clumpy and two-phase models, in order to search for polarisation diagnostics that can reveal structural properties of the torus. Secondly, we will compare these models to available observational data and investigate whether polarisation can be used as the ultimate tool to study the spatial distribution of the dust in AGN tori. This might also help in unravelling the properties and chemical composition of the dust, which plays a role in shaping the observed SED, in particular with respect to the characteristics of the silicate features. Our ultimate goal is to generate a self-consistent coupling of SKIRT and STOKES, which will allow us to coherently compute spectropolarimetric properties of AGNs from X-ray to submm wavelengths.

\section{Acknowledgements}

We gratefully acknowledge support from COST actions MP1104 "Polarisation as a tool to study Solar System and beyond" and MP0905 "Black Holes in a Violent Universe". This work benefitted from the CHARM framework (Contemporary physical challenges in Heliospheric and AstRophysical Models), a phase VII Interuniversity Attraction Pole (IAP) program organized by BELSPO, the BELgian federal Science Policy Office.

\section{References}

Antonucci, R. 1993, ARAESA 31, 473

Baes, M., Davies, J. I., Dejonghe, H., et al. 2003, MNRAS 343, 1081

Baes, M., Verstappen, J., De Looze, I., et al. 2011, ApJS 196, 22

Batcheldor, D., Robinson, A., Axon, D. J., et al. 2011, ApJ 738, 90

Camps, P. \& Baes, M. 2015, Astronomy and Computing 9, 20

Camps, P., Baes, M., \& Saftly, W. 2013, A\&A 560, A35

Dullemond, C. P. \& van Bemmel, I. M. 2005, A\&A 436, 47

Efstathiou, A. \& Rowan-Robinson, M. 1995, MNRAS 273, 649

Feltre, A., Hatziminaoglou, E., Fritz, J., \& Franceschini, A. 2012, MNRAS 426, 120

Fritz, J., Franceschini, A., \& Hatziminaoglou, E. 2006, MNRAS 366, 767

Goosmann, R. W., Gaskell, C. M., \& Marin, F. 2014, Advances in Space Research 54, 1341

Hönig, S. F., Beckert, T., Ohnaka, K., \& Weigelt, G. 2006, A\& A 452, 459

Marin, F. 2014, MNRAS 441, 551

Marin, F., Goosmann, R. W., Gaskell, C. M., Porquet, D., \& Dovčiak, M. 2012, A\& A 548, A121

Nenkova, M., Ivezić, Ž., \& Elitzur, M. 2002, ApJL 570, L9

Nenkova, M., Sirocky, M. M., Ivezić, Ž., \& Elitzur, M. 2008a, ApJ 685, 147 
Nenkova, M., Sirocky, M. M., Nikutta, R., Ivezić, Ž., \& Elitzur, M. 2008b, ApJ 685, 160

Popović, L. Č., Jovanović, P., Stalevski, M., et al. 2012, A\&A 538, A107

Saftly, W., Camps, P., Baes, M., et al. 2013, A\&A 554, A10

Saftly, W., Baes, M., \& Camps, P. 2014, A\&A 561, A77

Schartmann, M., Meisenheimer, K., Camenzind, M., Wolf, S., \& Henning, T. 2005, A\&3A 437, 861

Schartmann, M., Meisenheimer, K., Camenzind, M., et al. 2008, A\&A 482, 67

Schartmann, M., Wada, K., Prieto, M. A., Burkert, A., \& Tristram, K. R. W. 2014, MNRAS 445,3878

Smith, J. E., Young, S., Robinson, A., et al. 2002, MNRAS 335, 773

Stalevski, M. 2012, Bulgarian Astronomical Journal 18, 3

Stalevski, M., Fritz, J., Baes, M., Nakos, T., \& Popović, L. Č. 2012a, MNRAS 420, 2756

Stalevski, M., Jovanović, P., Popović, L. Č., \& Baes, M. 2012b, MNRAS 425, 1576

Urry, C. M. \& Padovani, P. 1995, PASP 107, 803

Wada, K., Papadopoulos, P. P., \& Spaans, M. 2009, ApJ 702, 63 